\title{
Les outils de mesure de la qualité de vie sont-ils pertinents ?
}

\section{Are tools for measuring quality of life relevant?}

\author{
M. Schmidt • E. Azoulay \\ (C) SRLF et Springer-Verlag France 2013
}

\section{Introduction}

Les résultats de la réanimation se sont longtemps limités à la survie quelques semaines après le début de la maladie aiguë. La réanimation prenait alors en effet en charge des patients relativement jeunes, avec peu de comorbidités, qui s'ils survivaient à la maladie aiguë, tout laissait penser qu'a priori, ils reviendraient à la vie qu'ils avaient avant. Ainsi, jusque récemment, le pronostic à long terme ou la qualité de vie (QOL) des patients sortis vivants de réanimation étaient négligés. Certes, sortir vivant de réanimation reste un premier succès légitime pour les médecins et infirmiers ayant pris en charge le patient. Cependant, la prise de conscience que la réanimation pouvait laisser des séquelles physiques, psychiques ou cognitives s'est imposée aux réanimateurs. Ces séquelles physiques (lourdeurs et raideurs articulaires, troubles de la marche, difficultés respiratoires, insuffisance rénale résiduelle, etc.) et mentales (symptômes d'anxiété, de stress ou de dépression) de la réanimation sont souvent intriquées avec des séquelles cognitives (état de stress post-traumatique). De plus, beaucoup de patients décrivent une diminution de leur QOL physique ou mentale après un séjour en réanimation.

\section{La qualité de vie : une valeur qualitative mesurée par une échelle de mesure}

Sans pouvoir établir avec certitude de relation de causalité entre les séquelles physiques, psychiques et cognitives,

M. Schmidt $(\bowtie)$

Université Paris-VI-Pierre-et-Marie-Curie, F-75013 Paris, France

e-mail : matthieuschmidt@yahoo.fr

Service de réanimation médicale, groupe hospitalier Pitié-Salpêtrière,

Assistance publique-Hôpitaux de Paris, France

E. Azoulay

Service de réanimation médicale,

Assistance publique-Hôpitaux de Paris,

hôpital Saint-Louis, France

Université Paris-VII, Paris, France d'une part, et l'altération de la QOL, d'autre part, il est apparu logique de ne plus s'intéresser uniquement à la survie, mais aussi à des critères de jugement plus qualitatifs comme la QOL des survivants [1,2]. Cependant, un des paradoxes de la mesure de la QOL est d'évaluer une valeur qualitative par des échelles quantitatives. Le concept de QOL est en effet subjectif, multidimensionnel et dépendant des valeurs socioculturelles des individus [3]. La QOL à un instant donné dépend aussi beaucoup de la QOL basale. Pour gérer cette complexité, il est nécessaire d'avoir des outils fiables, reproductibles et faciles d'utilisation. Il est aussi préférable de mieux définir de façon globale les conséquences physiques et psychologiques d'un séjour en réanimation, pour alors mieux informer les patients et leurs familles.

\section{Qualité de vie après la réanimation : des groupes de malades hétérogènes}

Cependant, les outils actuellement disponibles pour évaluer la QOL après la réanimation ont des limites méthodologiques. L'évaluation de la QOL liée à la santé se restreint à la description des répercussions d'une maladie ou de ses traitements dans les domaines physique, psychologique, relationnel ou symptomatique. Ainsi, les échelles les plus fréquemment utilisées (Short-form 36 ; EuroQol-5D) n'intègrent que certaines dimensions de la QOL et doivent être interprétées en fonction du contexte et de la pathologie aiguë ou chronique du patient. Cela est particulièrement vrai en réanimation, où la variété des diagnostics et les horizons divers des patients (médicaux, chirurgicaux, comorbidités...) ne permettent pas de déterminer finement quelle est la QOL à la sortie de réanimation [4]. Tout au plus, l'évaluation de la QOL doit rester focalisée à un groupe spécifique de patients, identifiés par leurs motifs d'admission ou leurs comorbidités. L'âge, les comorbidités et la sévérité du patient à son admission sont des déterminants majeurs de la QOL [5]. Ainsi, toute pathologie chronique préexistante pourrait accentuer la détérioration de la QOL induite par le séjour en réanimation. Obtenir la mesure de la QOL à 
l'admission parait donc crucial mais n'est néanmoins pas une chose facile. En effet, l'état du patient à son admission ne lui permet généralement pas de remplir un questionnaire. Par ailleurs, la famille du patient peut renseigner sa QOL avant la réanimation, mais la fiabilité de cette mesure est encore controversée [6,7]. Cette information reste judicieuse et utile. Elle permet de mieux définir l'impact du séjour en réanimation sur la QOL et d'orienter les cliniciens vers l'octroi d'un projet thérapeutique protégeant cette QOL. Par ailleurs, le fait que la QOL à l'admission en réanimation soit associée à la mortalité [8] permet d'encourager les réanimateurs à respecter les directives prévisionnelles des patients ainsi que les demandes de la famille pour les patients ayant une QOL préalablement altérée. Mettre en avant la QOL telle que perçue par la famille permet aussi aux proches d'adhérer au projet thérapeutique, en le justifiant avant tout par l'état préalable du patient, ses préférences et ses valeurs.

\section{La réanimation affecte la qualité de vie}

Une amélioration de la QOL après la sortie de réanimation pourrait être expliquée par une meilleure récupération et adaptation du patient à son état et non par une réelle récupération physique ou psychique [9]. De plus, les symptômes de stress, d'anxiété ou de dépression réduisent également la QOL. Il est donc nécessaire d'interpréter la QOL au regard des scores d'anxiété, de dépression et du risque de stress post-traumatique. Malgré un important corpus de publications [8-10] et des outils d'évaluation pertinents, la mesure de la QOL des patients après leur sortie de réanimation n'est toujours pas une pratique clinique courante. Il devient alors important d'utiliser ces outils pour définir et évaluer des stratégies interventionnelles qui intègrent une réhabilitation psychologique et physique précoce, afin de diminuer l'impact négatif de la réanimation sur la QOL. Des études ayant pour objectif principal l'amélioration de la QOL des survivants à la réanimation doivent intégrer l'ensemble des symptômes présents chez ces patients avec pour but ultime de réduire la pénibilité d'une survie qui est aujourd'hui l'issue de loin la plus fréquente chez les patients admis en réanimation.

Conflit d'intérêt : les auteurs déclarent ne pas avoir de conflit d'intérêt.

\section{Références}

1. Ferguson ND, Scales DC, Pinto R, et al (2013) Integrating mortality and morbidity outcomes: using quality-adjusted life years in critical care trials. Am J Respir Crit Care Med 187:256-61

2. Hunt SM, McEwen J (1980) The development of a subjective health indicator. Sociol Health Illn 2:231-46

3. Study protocol for the World Health Organization project to develop a Quality of Life assessment instrument (WHOQOL) (1993) Qual Life Res 2:153-9

4. Black NA, Jenkinson C, Hayes JA, et al (2001) Review of outcome measures used in adult critical care. Crit Care Med 29:2119-24

5. Abelha FJ, Santos CC, Maia PC, et al (2007) Quality of life after stay in surgical intensive care unit. BMC Anesthesiol 7:8

6. Capuzzo M, Grasselli C, Carrer S, et al (2000) Quality of life before intensive care admission: agreement between patient and relative assessment. Intensive Care Med 26:1288-95

7. Hofhuis J, Hautvast JL, Schrijvers AJ, et al (2003) Quality of life on admission to the intensive care: can we query the relatives? Intensive Care Med 29:974-9

8. Hofhuis JG, Spronk PE, van Stel HF, et al (2007) Quality of life before intensive care unit admission is a predictor of survival. Crit Care 11:R78

9. Sprangers MA, Schwartz CE (1999) Integrating response shift into health-related quality of life research: a theoretical model. Soc Sci Med 48:1507-15

10. Wehler M, Geise A, Hadzionerovic D, et al (2003) Health-related quality of life of patients with multiple organ dysfunction: individual changes and comparison with normative population. Crit Care Med 31:1094-101 